QUARTERLY OF APPLIED MATHEMATICS

VOLUME LXVII, NUMBER 3

SEPTEMBER 2009, PAGES 553-558

S 0033-569X(09)01136-8

Article electronically published on June 9, 2009

\title{
POLYNOMIAL DECAY TO THERMOELASTIC PLATES WITH MEMORY
}

\author{
BY \\ PEDRO GAMBOA ROMERO \\ Institute of Mathematics, Universidade Federal do Rio de Janeiro, Av. Brigadeiro Trompowski s/n, \\ Caixa Postal 68530 CEP:21945-970.RJ., Brazil
}

Abstract. We consider the linear model of thermoelastic plates with memory and we show that the solution decays polynomially with rates that depend on the regularity of the initial data.

1. Introduction. Let $\Omega$ be an open bounded set of $\mathbb{R}^{n}$ with smooth boundary $\Gamma$. Here we consider the transverse oscillations of thermoelastic plate configurates over $\Omega$. Denoting by $u$ and $\theta$ the vertical deflection and the temperature of the plate, the model that defines the oscillations of the plate is given by

$$
\begin{aligned}
& \left.\varrho u_{t t}+\gamma \Delta^{2} u+m \Delta \theta=0 \quad \text { in } \Omega \times\right] 0,+\infty[\text {, } \\
& \left.c \theta_{t}-\int_{0}^{\infty} \kappa(s) \Delta \theta(t-s) d s-m \Delta u_{t}=0 \quad \text { in } \quad \Omega \times\right] 0,+\infty[.
\end{aligned}
$$

We consider Dirichlet or clamped boundary conditions for $u$ and a Dirichlet boundary condition for $\theta$ that is

$$
u=\Delta u=\theta=0 \quad \text { in } \quad \partial \Omega \times] 0,+\infty[
$$

or

$$
\left.u=\frac{\partial u}{\partial \nu}=\theta=0 \quad \text { in } \quad \partial \Omega \times\right] 0,+\infty[
$$

and has initial value

$$
\begin{array}{cl}
u(0)=u^{0}, \quad u^{\prime}(0)=u^{1}, \quad \theta(0)=\theta^{0} & \text { in } \Omega, \\
\theta(-s)=\psi(s) & \text { in } \Omega \times] 0,+\infty[
\end{array}
$$

Received March 1, 2008.

2000 Mathematics Subject Classification. Primary 35M10, 35B30.

Key words and phrases. Thermoelastic plates, polynomial decay, materials with memory.

E-mail address: pgamboa@im.ufrj.br 
where $u^{0}, u^{1}, \theta^{0}$ and $\psi$ are given functions and $\kappa:[0,+\infty[\rightarrow \mathbb{R}$ is the memory kernel. Denoting by $\mu(s)=-\kappa^{\prime}(s)$, the hypotheses we impose on $\mu$ are the following:

$$
\begin{gathered}
\mu \in \mathcal{C}^{1}\left(\mathbb{R}^{+}\right) \cap H^{1}\left(\mathbb{R}^{+}\right), \quad \mu(s) \geq 0, \quad \mu^{\prime}(s) \leq 0 \quad \forall s \in \mathbb{R}^{+}, \\
\mu^{\prime}(s)+\sigma \mu(s) \leq 0 .
\end{gathered}
$$

Here we follow the same notations as in $[5$. In that article the authors proved that the system is not exponentially stable. By using the La Salle principle, the authors showed that the solution in general goes to zero but they did not report any rate of decay. The main result of this paper is to show that there exists a polynomial rate of decay of the solution that can be improved by improving the regularity of the initial data. We consider both clamped and Dirichlet boundary conditions for the displacement $u$.

2. Functional spaces and notations. Let us denote by $\mathcal{H}$ the Hilbert space with inner product $\langle\cdot, \cdot\rangle_{\mathcal{H}}$ and let us denote by $\|\cdot\|_{\mathcal{H}}$ the induced norm of $\mathcal{H}$. Let us introduce the history space

$$
\mathcal{M}_{1}=L_{\mu}^{2}\left(0,+\infty ; H_{0}^{1}(\Omega)\right)
$$

which is a Hilbert space. Let us denote by $\mathfrak{T}: \mathcal{M}_{1} \rightarrow \mathcal{M}_{1}$ the operator given by

$$
\mathfrak{T} \eta=-\eta_{s} \quad \eta \in \mathcal{D}(\mathfrak{T})=\left\{\eta \in \mathcal{M}_{1} ; \eta_{s} \in \mathcal{M}_{1} \quad \text { and } \quad \eta(0)=0\right\},
$$

with

$$
\langle\mathfrak{T} \eta, \eta\rangle_{\mathcal{M}_{1}}=\int_{0}^{\infty} \mu^{\prime}(s)\|\nabla \eta(s)\|^{2} d s \leq 0 \quad \forall \eta \in \mathcal{D}(\mathfrak{T}) .
$$

Let us introduce the summed past history of $\theta$ as

$$
\eta^{t}(s)=\int_{0}^{s} \theta(t-y) d y \quad(t, s) \in \mathbb{R}^{+} \times \mathbb{R}^{+} .
$$

It is not difficult to see that $\eta$ satisfies

$$
\eta_{t}^{t}+\eta_{s}=\theta \quad t \in \mathbb{R}^{+} .
$$

Moreover

$$
\eta^{0}(s)=\int_{0}^{s} \psi(y) d y \quad s \in \mathbb{R}^{+} \text {in } \quad \Omega, \quad \eta^{t}(0)=0 \quad \forall t \geq 0 .
$$

Denoting by $z(t)=\left(u(t), v(t), \theta(t), \eta^{t}\right)^{T}, z^{0}=\left(u^{0}, v^{0}, \theta^{0}, \eta^{0}\right)^{T}$ in $\mathcal{H}$, the semigroup formulation of the system (1.1)-(1.6) is given by

$$
\left\{\begin{array}{l}
\frac{d}{d t} z(t)=\mathcal{L} z(t) \\
z(0)=z^{0}
\end{array}\right.
$$

where $\mathcal{L}: D(\mathcal{L}) \subset \mathcal{H} \rightarrow \mathcal{H}$ is defined as

$$
\mathcal{L}\left(\begin{array}{c}
u \\
v \\
\theta \\
\eta
\end{array}\right)=\left(\begin{array}{c}
v \\
-\frac{1}{\rho} \Delta(\gamma \Delta u+m \theta) \\
+\frac{m}{c} \Delta v+\frac{1}{c} \int_{0}^{\infty} \mu(s) \Delta \eta(s) d s \\
\theta+\mathfrak{T} \eta
\end{array}\right)
$$


with domain

$$
\mathcal{D}(\mathcal{L})=\left\{z \in \mathcal{H}_{D} ; \gamma \Delta u+m \theta, v \in H_{0}^{1} \cap H^{2}, \theta \in H_{0}^{1}, \mu * \Delta \eta \in L^{2}, \eta \in \mathcal{D}(\mathfrak{T})\right\}
$$

or

$$
\mathcal{D}(\mathcal{L})=\left\{z \in \mathcal{H}_{C} ; \gamma \Delta u+m \theta \in H^{2}, v \in H_{0}^{2}, \theta \in H_{0}^{1}, \mu * \Delta \eta \in L^{2}, \eta \in \mathcal{D}(\mathfrak{T})\right\},
$$

where $\mathcal{H}_{C}=H_{0}^{2} \times L^{2} \times L^{2} \times \mathcal{M}_{1}$ and $\mathcal{H}_{D}=H_{0}^{1} \cap H^{2} \times L^{2} \times L^{2} \times \mathcal{M}_{1}$. From now on, we denote by $\mathcal{H}$ any of the spaces $\mathcal{H}_{D}$ or $\mathcal{H}_{C}$.

Theorem 2.1. The operator $\mathcal{L}$ is the infinitesimal generator of a $C_{0}$ semigroup of contractions over $\mathcal{H}$ that we denote as $S(t)$.

Proof. See [5].

In particular we have that problem (2.5) is well posed in the corresponding semigroup spaces.

3. Decay of solutions. In this section we study the polynomial decay of energy to the system

$$
\begin{aligned}
\varrho u_{t t}+\gamma \Delta^{2} u+m \Delta \theta & =0, \\
c \theta_{t}-\int_{0}^{\infty} \mu(s) \Delta \eta(s) d s-m \Delta u_{t} & =0, \\
\eta_{t}+\eta_{s} & =\theta,
\end{aligned}
$$

with boundary conditions (1.3) or (1.4) and initial condition (1.5)-(1.6). To facilitate our analysis we introduce the following notation:

$$
\begin{gathered}
(\mu * \eta)(t)=\int_{0}^{\infty} \mu(s) \eta^{t}(s) d s, \\
(\mu \square \nabla \eta)(t)=\int_{0}^{\infty} \mu(s)\left|\nabla \eta^{t}(s)\right|^{2} d s .
\end{gathered}
$$

Let us define the first- and second-order energy

$$
\begin{gathered}
\mathbf{E}_{1}(t)=\frac{1}{2} \int_{\Omega}\left\{\varrho\left|u_{t}(t)\right|^{2}+\gamma|\Delta u(t)|^{2}+c|\theta(t)|^{2}+(\mu \square \nabla \eta)(t)\right\} d x, \\
\mathbf{E}_{2}(t)=\frac{1}{2} \int_{\Omega}\left\{\varrho\left|u_{t t}(t)\right|^{2}+\gamma\left|\Delta u_{t}(t)\right|^{2}+c\left|\theta_{t}(t)\right|^{2}+\left(\mu \square \nabla \eta_{t}\right)(t)\right\} d x .
\end{gathered}
$$

Using the usual multiplicative techniques we can show that

$$
\begin{aligned}
& \frac{d}{d t} \mathbf{E}_{1}(t)=\int_{0}^{\infty} \mu^{\prime}(s) \int_{\Omega}|\nabla \eta(s)|^{2} d x d s \\
& \frac{d}{d t} \mathbf{E}_{2}(t)=\int_{0}^{\infty} \mu^{\prime}(s) \int_{\Omega}\left|\nabla \eta_{t}(s)\right|^{2} d x d s .
\end{aligned}
$$

Let us take $q_{k} \in C^{2}$ such that $q_{k}=\nu_{k}$ on $\Gamma$. Let us denote by $J_{0}$ the functional

$$
J_{0}(t)=\int_{\Omega} \varrho u_{t} q_{k} \frac{\partial u}{\partial x_{k}} d x+\frac{1}{\mu_{0}} \int_{\Omega}[c \theta-m \Delta u] q_{k} \frac{\partial \mu * \eta}{\partial x_{k}} .
$$

The next Lemma is only necessary when we use clamped boundary conditions. 
Lemma 3.1. If $u$ and $\theta$ satisfy the boundary conditions (1.4), then there exists a positive constant $C$ for which we have

$$
\frac{d}{d t} J_{0}(t) \leq-\frac{\gamma}{2} \int_{\Gamma}|\Delta u|^{2} d \Gamma+C E(t) .
$$

Proof. Multiplying equation (3.1) by $q_{k} \frac{\partial u}{\partial x_{k}}$ and equation (3.2) by $\frac{1}{\mu_{0}} q_{k} \frac{\partial \mu * \eta}{\partial x_{k}}$, summing the product result, and using similar arguments as in 2], our conclusion follows.

Let us define the functional $J$ as

$$
J(t)=-N_{1} \int_{\Omega} \theta(\mu * \eta)+\nabla u(\mu * \nabla \eta) d x-\int_{\Omega} \theta p_{t} d x+\frac{1}{4} \int_{\Omega} u u_{t} d x+\frac{2 \delta}{\gamma} J_{0}(t) .
$$

Lemma 3.2. Let us suppose that $u$ and $\theta$ satisfy (1.4). Then there exist positive constants for which we have

$$
\frac{d}{d t} J(t) \leq-\frac{N \mu_{0}}{2} \int_{\Omega}|\theta|^{2} d x-\frac{1}{2} \int_{\Omega}\left|u_{t}\right|^{2} d x .
$$

Proof. Multiplying equation (3.2) by $\mu * \eta$ we get

$$
\int_{\Omega} \theta_{t}(\mu * \eta)(t) d x+\int_{\Omega}|\mu * \nabla \eta|^{2} d x+\int_{\Omega} u_{t} \mu * \Delta \eta d x=0 .
$$

Using

$$
\frac{d}{d t} \int_{\Omega} \theta \mu * \eta d x=\int_{\Omega} \theta_{t} \mu * \eta d x+\int_{\Omega} \theta\left(\mu * \eta_{t}\right) d x
$$

we get from (3.6) and (3.7) that

$$
\frac{d}{d t} \int_{\Omega} \theta \mu * \eta=-\int_{\Omega}|\mu * \nabla \eta|^{2} d x-\int_{\Omega} u_{t} \mu * \Delta \eta d x+\int_{\Omega} \theta\left(\mu * \eta_{t}\right) d x .
$$

Note that

$$
-\int_{\Omega} u_{t} \mu * \Delta \eta d x=\frac{d}{d t} \int_{\Omega} \nabla u(\mu * \nabla \eta) d x-\int_{\Omega} \nabla u\left(\mu * \nabla \eta_{t}\right) d x .
$$

From here we have

$$
\begin{aligned}
& \frac{d}{d t} \underbrace{\left\{\int_{\Omega} \theta \mu * \eta+\nabla u(\mu * \nabla \eta) d x\right\}}_{:=-F(t)} \\
& \quad=-\int_{\Omega}|\mu * \nabla \eta|^{2} d x-\int_{\Omega} \nabla u\left(\mu * \nabla \eta_{t}\right) d x+\int_{\Omega} \theta\left(\mu * \eta_{t}\right) d x .
\end{aligned}
$$

Substituting equations (3.3) into (3.8) and using

$$
\int_{\Omega}|\mu * \nabla \eta|^{2} d x \leq \mu_{0} \int_{\Omega} \mu \square \nabla \eta d x
$$

we get

$$
\frac{d}{d t} F(t) \leq-\frac{\mu_{0}}{2} \int_{\Omega}|\theta|^{2} d x+C_{\epsilon} \int_{\Omega} \mu \square \nabla \eta+\mu \square \nabla \eta_{t} d x+\epsilon \int_{\Omega}|\nabla u|^{2} .
$$

Let us introduce the following multipliers:

$$
\Delta \omega=\theta, \quad \Delta p=u, \quad \text { in } \quad \Omega, \quad p=\theta=0, \quad \text { on } \quad \Gamma .
$$


Multiplying equation (3.2) by $p_{t}$ defined above, we get

$$
\begin{aligned}
\frac{d}{d t} \int_{\Omega} \theta p_{t} & =\int_{\Omega} \theta_{t} p_{t}+\int_{\Omega} \theta p_{t t} \\
& =\int_{\Omega}\left(\mu * \Delta \eta+\Delta u_{t}\right) p_{t}+\int_{\Omega} \Delta \omega p_{t t} \\
& =\int_{\Omega}\left|u_{t}\right|^{2}+\int_{\Omega}(\mu * \eta) u_{t}+\int_{\Omega} \omega\left(-\Delta^{2} u-\Delta \theta\right) \\
& =\int_{\Omega}\left|u_{t}\right|^{2}+\int_{\Omega}(\mu * \eta) u_{t}+\int_{\Gamma} \frac{\partial \omega}{\partial \nu} \Delta u d \Gamma-\int_{\Omega} \theta \Delta u-\int_{\Omega}|\theta|^{2} .
\end{aligned}
$$

Note that for any $\epsilon>0$ there exists $C_{\epsilon}>0$ for which we have

$$
\left|\int_{\Omega}(\mu * \eta) u_{t}\right| \leq \epsilon \int_{\Omega}\left|u_{t}(t)\right|^{2}+C_{\epsilon} \int_{\Omega} \mu \square \nabla \eta d x
$$

and

$$
\left|\int_{\Omega} \theta \Delta u\right| \leq C_{\epsilon} \int_{\Omega}|\theta(t)|^{2} d x+\epsilon \int_{\Omega}|\Delta u(t)|^{2} d x
$$

Since the normal derivative of $\omega$ is bounded by the $L^{2}$-norm of $\theta$, from inequalities (3.12), (3.13), and (3.14), we get

$$
\begin{aligned}
-\frac{d}{d t} \int_{\Omega} \theta p_{t} \leq & -\frac{1}{2} \int_{\Omega}\left|u_{t}(t)\right|^{2} d x+c_{\epsilon \delta} \int_{\Omega}|\theta(t)|^{2} d x \\
& +\epsilon \int_{\Omega}|\Delta u(t)|^{2}+\delta \int_{\Gamma}|\Delta u(t)|^{2}+C_{\epsilon} \int_{\Omega} \mu \square \nabla \eta d x .
\end{aligned}
$$

Multiplying equation (3.1) by $u$ we get

$$
\begin{aligned}
\frac{d}{d t} \int_{\Omega} u u_{t} d x & =\int_{\Omega}\left|u_{t}(t)\right|^{2} d x+\int_{\Omega} u \cdot u_{t t} d x \\
& =\int_{\Omega}\left|u_{t}(t)\right|^{2} d x+\int_{\Omega} u\left(-\Delta^{2} u-\Delta \theta\right) d x \\
& \leq \int_{\Omega}\left|u_{t}(t)\right|^{2} d x-\frac{1}{2} \int_{\Omega}|\Delta u|^{2}+\frac{1}{2} \int_{\Omega}|\theta|^{2} d x
\end{aligned}
$$

From (3.15) and (3.16) we get

$$
\begin{aligned}
-\frac{d}{d t}\left\{\int_{\Omega} \theta p_{t}-\frac{1}{4} \int_{\Omega} u u_{t} d x\right\} \leq & -\frac{1}{8} \int_{\Omega}\left|u_{t}(t)\right|^{2}+|\Delta u(t)|^{2} d x \\
& +c_{\epsilon} \int_{\Omega}|\theta(t)|^{2} d x+C_{\epsilon} \int_{\Omega} \mu \square \nabla \eta d x+\delta \int_{\Gamma}|\Delta u(t)|^{2} .
\end{aligned}
$$

Recalling the definition of $J$ and taking $\epsilon$ and $\delta$ small enough, we conclude that there exists $\gamma_{0}>0$ for which we have

$$
\frac{d}{d t} J(t) \leq-\gamma_{0} \mathbf{E}(t)+c_{\epsilon} \int_{\Omega} \mu \square \nabla \eta+\mu \square \nabla \eta_{t} d x .
$$

In the case where the boundary condition (1.3) holds, Lemma 3.2 also holds easily because in this case we have no boundary terms to estimate. We are now ready to prove the main result of this paper. 
Theorem 3.1. Let us take initial data $z_{0} \in D(\mathcal{L})$. Then there exists a positive constant $C$ for which we have

$$
\left\|S(t) z_{0}\right\|_{\mathcal{H}} \leq \frac{C}{\sqrt{t}}\left\|z_{0}\right\|_{D(\mathcal{L})} .
$$

Moreover for any $m \in \mathbb{N}$, we get

$$
\left\|S(t) z_{0}\right\|_{\mathcal{H}} \leq \frac{C}{t^{m / 2}}\left\|z_{0}\right\|_{D\left(\mathcal{L}^{m}\right)} .
$$

Proof. Taking $\mathfrak{F}(t)=N \mathbf{E}(t)+N \mathbf{E}_{2}(t)+J(t)$, for $N$ large enough, we get $\mathfrak{F}(t) \geq 0$ and also,

$$
\frac{d}{d t} \mathfrak{F}(t) \leq-\gamma_{0} \mathbf{E}(t) \quad \Rightarrow \quad \gamma_{0} \int_{0}^{\infty} \mathbf{E}(t) d t \leq \mathfrak{F}(0) \leq C\left(\mathbf{E}(0)+\mathbf{E}_{2}(0)\right) .
$$

From here we get

$$
\mathbf{E}(t) \leq C \frac{\left\{\mathbf{E}(0)+\mathbf{E}_{2}(0)\right\}}{t} .
$$

So we get (3.18) (not optimal). Note that (3.18) implies

$$
\left\|S(t) \mathcal{L}^{-1} z_{0}\right\|_{\mathcal{H}} \leq \frac{C}{\sqrt{t}}\left\|z_{0}\right\|_{\mathcal{H}} \Rightarrow\left\|s(t) \mathcal{L}^{-1}\right\| \leq \frac{C}{\sqrt{t}} .
$$

Therefore to get (3.19) we consider

$$
\left\|S(t) \mathcal{L}^{-m}\right\|=\left\|S\left(\frac{t}{m}\right)^{m} \mathcal{L}^{-m}\right\| \leq\left\|S\left(\frac{t}{m}\right) \mathcal{L}^{-1}\right\|^{m} \leq C^{m}\left[\left(\frac{t}{m}\right)^{-1 / 2}\right]^{m} .
$$

From here our conclusion follows.

Acknowledgment. The author would like to thank Professor Jaime Muñoz Rivera for fruitful discussions about the problem.

\section{REFERENCES}

[1] M. Fabrizio, B. Lazzari, J. E. Muñoz Rivera, Asymptotic behaviour of thermoelastic plates of weakly hyperbolic type, Differential Integral Equations 13 (2000) 1347-1370. MR.1787071 (2001h:74031)

[2] C. Giorgi, V. Pata, Stability of linear thermoelastic systems with memory, Math. Models Methods Appl. Sci 11 (2001) 627-644. MR1832996 (2002e:74011)

[3] Maurizio Grasselli, J. E. Muñoz Rivera, V. Pata, On the energy decay of the linear thermoelastic plate with memory, J. Math. Anal. Appl. 309 (2005) 1-14. MR.2154022 (2006c:35281)

[4] M. E. Gurtin, A. C. Pipkin, A general theory of heat conduction with finite wave speeds, Arch. Ration. Mech. Anal 31 (1968) 113-126. MR1553521

[5] J. E. Muñoz Rivera, M. G. Naso, E. Vuk, Asymptotic behavior of the energy for electromagnetic systems with memory, Math. Methods. Appl. Sci. 7 (2004) 819-841. MR2055321 (2005a:35268)

[6] J. E. Muñoz Rivera, R. Racke, Smoothing properties, decay and global existence of solutions to nonlinear coupled systems of thermoelastic type, SIAM J. Math. Anal. 26 (1995) 1547-1563. MR.1356459 (96h:35217)

[7] V. Pata, A. Zucchi, Attractors for a damped hyperbolic equation with linear memory, Adv. Math. Sci. Appl. 11 (2001) 505-529. MR 1907454(2003f:35027)

[8] A. Pazy, Semigroups of Linear Operators and Applications to Partial Differential Equations, SpringerVerlag, New York, 1983. MR710486 (85g:47061)

[9] J. Pruss, On the spectrum of $C_{0}$-semigroups, Trans. Amer. Math. Soc. 284 (1984) 847-857. MR743749 (85f:47044) 